\title{
Common Fixed Point Theorems Using the E.A. and CLR Properties in 2-Menger Spaces
}

\author{
Balbir Singh, ${ }^{1}$ Vishal Gupta, ${ }^{2}$ and Sanjay Kumar ${ }^{3}$ \\ ${ }^{1}$ Department of Mathematics, B.M. Institute of Engineering and Technology, Sonipat-131001 Haryana, India \\ ${ }^{2}$ Department of Mathematics, M.M. University, Mullana, Ambala-133203 Haryana, India \\ ${ }^{3}$ Department of Mathematics, D.C.R. University of Science and Technology, Murthal, Sonipat-131039 Haryana, India
}

Correspondence should be addressed to Sanjay Kumar; sanjuciet@rediffmail.com

Received 31 January 2013; Revised 2 April 2013; Accepted 4 April 2013

Academic Editor: Chuanxi Qian

Copyright (C) 2013 Balbir Singh et al. This is an open access article distributed under the Creative Commons Attribution License, which permits unrestricted use, distribution, and reproduction in any medium, provided the original work is properly cited.

First, we prove a common fixed point theorem using weakly compatible maps in 2-Menger space with $t$-norm of Hadzic type. Second, we prove a common fixed point theorem using the E.A. property along with weakly compatible maps. Further, we obtained a common fixed point theorem using the CLR property along with weakly compatible maps. At the end, we provide an application of our main theorem for four finite families of mappings.

\section{Introduction}

The theory of probabilistic metric spaces is an important part of stochastic analysis, and so it is of interest to develop the fixed point theory in such spaces. The first result from the fixed point theory in probabilistic metric spaces was obtained by Sehgal and Bharucha-Reid [1]. Since then many fixed points theorems for single valued and multivalued mappings in probabilistic metric spaces have been proved in [2-8]. The study of 2-metric spaces was initiated by Gähler [9] and some fixed point theorems in 2-metric spaces were proved in [2, 8, 10-13]. In 1987, Zeng [14] gave the generalization of 2metric to probabilistic 2-metric as follows.

A probabilistic 2-metric space is an ordered pair $(X, F)$, where $X$ is an arbitrary set and $F$ is a mapping from $X^{3}$ into the set of distribution functions. The distribution function $F_{x, y, z}(t)$ will denote the value of $F_{x, y, z}$ at the positive real number $t$. The function $F_{x, y, z}$ is assumed to satisfy the following conditions:

(i) $F_{x, y, z}(0)=0$ for all $x, y, z \in X$;

(ii) $F_{x, y, z}(t)=1$ for all $t>0$ if and only if at least two of the three points $x, y, z$ are equal;

(iii) for distinct points $x, y \in X$, there exists a point $z \in X$ such that $F_{x, y, z}(t) \neq 1$ for $t>0$; (iv) $F_{x, y, z}(t)=F_{y, z, x}(t)=F_{z, x, y}(t)=\cdots$ for all $x, y, z \in$ $X$ and $t>0$;

(v) if $F_{x, y, w}\left(t_{1}\right)=1, F_{x, w, z}\left(t_{2}\right)=1$, and $F_{w, y, z}\left(t_{3}\right)=1$, then $F_{x, y, z}\left(t_{1}+t_{2}+t_{3}\right)=1$ for all $x, y, z, w \in X$ and $t_{1}, t_{2}, t_{3}>0$.

In 2003, Shi et al. [15] gave the notion of $n$th order $t$-norm as follows.

Definition 1. A mapping $\Delta: \prod_{i=1}^{n}[0,1] \rightarrow[0,1]$ is called an $n$th order $t$-norm if the following conditions are satisfied:

(i) $\Delta(0,0, \ldots, 0)=0, \Delta(a, 1,1, \ldots, 1)=a$ for all $a \in$ $[0,1]$

(ii) $\Delta\left(a_{1}, a_{2}, a_{3}, \ldots, a_{n}\right)=\Delta\left(a_{2}, a_{1}, a_{3}, \ldots, a_{n}\right)=\Delta\left(a_{2}, a_{3}\right.$, $\left.a_{1}, \ldots, a_{n}\right)=\cdots=\Delta\left(a_{2}, a_{3}, a_{4}, \ldots, a_{n}, a_{1}\right)$;

(iii) $a_{i} \geq b_{i}, i=1,2,3 \ldots, n$, implies $\Delta\left(a_{1}, a_{2}, a_{3}, \ldots, a_{n}\right) \geq$ $\Delta\left(b_{1}, b_{2}, b_{3}, \ldots, b_{n}\right)$;

(iv) $\Delta\left(\Delta\left(a_{1}, a_{2}, a_{3}, \ldots, a_{n}\right), b_{2}, b_{3}, \ldots, b_{n}\right)$

$=\Delta\left(a_{1}, \Delta\left(a_{2}, a_{3}, \ldots, a_{n}, b_{2}\right), b_{3}, \ldots b_{n}\right)$

$=\Delta\left(a_{1}, a_{2}, \Delta\left(a_{3}, a_{4}, \ldots, a_{n}, b_{2}, b_{3}\right), b_{4}, \ldots, b_{n}\right)$

$=\cdots=\Delta\left(a_{1}, a_{2}, a_{3}, \ldots, a_{n-1}, \Delta\left(a_{n}, b_{2}, b_{3}, \ldots, b_{n}\right)\right)$.

For $n=2$, we have a binary $t$-norm, which is commonly known as $t$-norm. 
Basic examples of $t$-norm are the Lukasiewicz $t$-norm $\Delta_{L}, \Delta_{L}(a, b)=\max (a+b-1,0), t$-norm $\Delta_{P}, \Delta_{P}(a, b)=a b$, and $t$-norm $\Delta_{M}, \Delta_{M}(a, b)=\min \{a, b\}$.

Definition 2 (see [6]). A special class of $t$-norms (called a Hadzic-type $t$-norm) is introduced as follows.

Let $\Delta$ be a $t$-norm and let $\Delta_{n}:[0,1] \rightarrow[0,1](n \in N)$ be defined in the following way:

$$
\begin{array}{r}
\Delta_{1}(x)=\Delta(x, x), \quad \Delta_{n+1}(x)=\Delta\left(\Delta_{n}(x), x\right) \\
(n \in N, x \in[0,1]) .
\end{array}
$$

We say that the $t$-norm $\Delta$ is of $H$ type if $\Delta$ is continuous and the family $\left\{\Delta_{n}(x), n \in N\right\}$ is equicontinuous at $x=1$.

The family $\left\{\Delta_{n}(x), n \in N\right\}$ is equicontinuous at $x=1$ if for every $\lambda \in(0,1)$ there exists $\delta(\lambda) \in(0,1)$ such that the following implication holds:

$$
x>1-\delta(\lambda) \quad \text { implies } \Delta_{n}(x)>1-\lambda \quad \forall n \in N .
$$

A trivial example of $t$-norm of $H$ type is $\Delta=\Delta_{M}$.

Remark 3. Every $t$-norm $\Delta_{M}$ is of Hadzic type but the converse need not be true; see [7].

There is a nice characterization of continuous $t$-norm.

(i) If there exists a strictly increasing sequence $\left\{b_{n}\right\}_{n \in N} \in$ $[0,1]$ such that $\lim _{n \rightarrow \infty} b_{n}=1$ and $\Delta\left(b_{n}, b_{n}\right)=b_{n}$ for all $n \in N$, then $\Delta$ is of Hadzic type.

(ii) If $\Delta$ is continuous and $\Delta$ is of Hadzic type, then there exists a sequence $\left\{b_{n}\right\}_{n \in N}$ as in (i).

Definition 4 (see [7]). If $\Delta$ is a $t$-norm and $\left(x_{1}, x_{2}\right.$, $\left.x_{3}, \ldots, x_{n}\right) \in[0,1]^{n}(n \in N)$, then $\Delta_{i=1}^{n} x_{i}$ is defined recurrely by 1 , if $n=1$ and $\Delta_{i=1}^{n} x_{i}=\Delta\left(\Delta_{i=1}^{n-1} x_{i}, x_{n}\right)$ for all $n \geq 2$. If $\left\{b_{n}\right\}_{n \in N}$ is a sequence of numbers from $[0,1]$, then $\Delta_{i=1}^{\infty} x_{i}$ is defined as $\lim _{n \rightarrow \infty} \Delta_{i=1}^{n} x_{i}$ (this limit always exists) and $\Delta_{i=1}^{\infty} x_{i}$ as $\Delta_{i=1}^{\infty} x_{n+i}$.

Definition 5. Let $X$ be any nonempty set and $D$ the set of all left-continuous distribution functions. A triplet $(X, F, \Delta)$ is said to be a 2-Menger space if the probabilistic 2-metric space $(X, F)$ satisfies the following condition:

$F_{x, y, z}(t) \geq \Delta\left(F_{x, y, w}\left(t_{1}\right), F_{x, w, z}\left(t_{2}\right), F_{w, y, z}\left(t_{3}\right)\right)$, where $t_{1}, t_{2}, t_{3}>0, t_{1}+t_{2}+t_{3}=t ; x, y, z, w \in X$; and $\Delta$ is the $3 r d$ order $t$-norm.

Definition 6. A sequence $\left\{x_{n}\right\}$ in a 2-Menger space $(X, F, \Delta)$ is said to be

(i) converge with limit $x$ if $\lim _{n \rightarrow \infty} F_{x_{n}, x, a}(t)=1$ for all $t>0$ and for every $a \in X$,

(ii) Cauchy sequence in $X$, if given $\epsilon>0, \lambda>0$, there exists a positive integer $N_{\epsilon, \lambda}$ such that

$$
F_{x_{n}, x_{m}, a}(\epsilon)>1-\lambda \quad \forall m, n>N_{\epsilon, \lambda} \text {, for every } a \in X,
$$

(iii) complete if every Cauchy sequence in $X$ is convergent in $X$.
In 1996, Jungck's [16] introduced the notion of weakly compatible as follows.

Definition 7. Two maps $f$ and $g$ are said to be weakly compatible if they commute at their coincidence points.

In 2002, Aamri and Moutawakil [17] generalized the notion of noncompatible mapping to the E.A. property. It was pointed out in [17] that the property E.A. buys containment of ranges without any continuity requirements besides minimizing the commutativity conditions of the maps to the commutativity at their points of coincidence. Moreover, the E.A. property allows replacing the completeness requirement of the space with a more natural condition of closeness of the range. Recently, some common fixed point theorems in probabilistic metric spaces/fuzzy metric spaces by the E.A. property under weak compatibility have been obtained in [18-20].

Definition 8 (see [17]). Let $f$ and $g$ be two self-maps of a metric $(X, d)$. The maps $f$ and $g$ are said to satisfy the E.A. property if there exists a sequence $\left\{x_{n}\right\}$ in $X$ such that

$$
\lim _{n \rightarrow \infty} f x_{n}=\lim _{n \rightarrow \infty} g x_{n}=u \quad \text { for some } u \in X .
$$

Now in a similar mode, we can state the E.A. property in 2Menger space as follows.

Definition 9. A pair of self-mappings $(f, g)$ of 2-Menger spaces $(X, F, \Delta)$ is said to hold the E.A. property if there exists a sequence $\left\{x_{n}\right\}$ in $X$ such that

$$
\lim _{n \rightarrow \infty} F_{f x_{n}, g x_{n}, p}(t)=1 \quad \forall t>0, p \in X .
$$

Example 10. Let $X=[0, \infty)$ be the usual metric space. Define $f, g: X \rightarrow X$ by $f x=x / 4$ and $g x=3 x / 4$ for all $x \in$ $X$. Consider the sequence $\left\{x_{n}\right\}=1 / n$. Since $\lim _{n \rightarrow \infty} f x_{n}=$ $\lim _{n \rightarrow \infty} g x_{n}=0$, then $f$ and $g$ satisfy the E.A. property.

Although E.A property is generalization of the concept of noncompatible maps, yet it requires either completeness of the whole space or any of the range spaces or continuity of maps. But on the contrary, the new notion of the CLR property (common limit range property) recently given by Sintunavarat and Kumam [21] does not impose such conditions. The importance of the CLR property ensures that one does not require the closeness of range subspaces.

Definition 11 (see [21]). Two maps $f$ and $g$ on 2-Menger spaces $X$ satisfy the common limit in the range of $g$ (CLRg) property if $\lim _{n \rightarrow \infty} f x_{n}=\lim _{n \rightarrow \infty} g x_{n}=g x$ for some $x \in X$.

Example 12. Let $X=[0, \infty)$ be the usual metric space. Define $f, g: X \rightarrow X$ by $f x=x+1$ and $g x=2 x$ for all $x \in X$. Consider the sequence $\left\{x_{n}\right\}=1+(1 / n)$. Since $\lim _{n \rightarrow \infty} f x_{n}=$ $\lim _{n \rightarrow \infty} g x_{n}=2=g 1, f$ and $g$ satisfy the CLRg property.

Now we state a lemma which is useful in our study.

Lemma 13 (see [22]). Let $(X, F, \Delta)$ be a 2-Menger space. If there exists $q \in(0,1)$ such that $F(x, y, z, q t) \geq F(x, y, z, t)$ for all $x, y, z \in X$ with $z \neq x, z \neq y$, and $t>0$, then $x=y$. 


\section{Weakly Compatible Maps}

Theorem 14. Let $(X, F, \Delta)$ be a complete 2-Menger space with continuous t-norm $\Delta$ of $H$ type. Let $S$ and $T$ be self-mappings of $X$. Then $S$ and $T$ have a unique common fixed point in $X$ if and only if there exist two self-mappings $A, B$ of $X$ satisfying the following:

(2.1) $A(X) \subset T(X)$ and $B(X) \subset S(X)$;

(2.2) the pairs $(A, S)$ and $(B, T)$ are weakly compatible;

(2.3) there exists $q \in(0,1)$ such that for every $x, y, p \in X$ and $t>0, F(A x, B y, p, q t) \geq \min \{F(S x, T y, p, t)$, $F(A x, S x, p, t), F(B y, T y, p, t), F(A x, T y, p, t)\} ;$

(2.4) one of the subsets $A(X), B(X), S(X)$, or $T(X)$ is a closed subset of $X$.

Indeed, $A, B, S$, and T have a unique common fixed point in $X$.

Proof. Suppose that $S$ and $T$ have a unique common fixed point, say $z \in X$.

Define $A: X \rightarrow X$ by $A x=z$ for all $x \in X$ and $B: X \rightarrow$ $X$ by $B x=z$ for all $x \in X$.

Then one can see that (2.1)-(2.4) are satisfied.

Conversely, assume that there exist two self-mappings $A, B$ of $X$ satisfying conditions (2.1)-(2.4). From condition (2.1) we can construct two sequences $\left\{x_{n}\right\}$ and $\left\{y_{n}\right\}$ of $X$ such that

$$
\begin{array}{r}
y_{2 n-1}=T x_{2 n-1}=A x_{2 n-2}, \quad y_{2 n}=S x_{2 n}=B x_{2 n-1} \\
\text { for } n=1,2, \ldots
\end{array}
$$

Putting $x=x_{2 n}$ and $y=x_{2 n+1}$ in (2.3), we have that for all $p \in X$ and $t>0$

$$
\begin{array}{r}
F\left(y_{2 n+1}, y_{2 n+2}, p, q t\right) \\
=F\left(A x_{2 n}, B x_{2 n+1}, p, q t\right) \\
\geq \min \left\{F\left(S x_{2 n}, T x_{2 n+1}, p, t\right),\right. \\
F\left(A x_{2 n}, S x_{2 n}, p, t\right), \\
F\left(B x_{2 n+1}, T x_{2 n+1}, p, t\right), \\
\left.F\left(A x_{2 n}, T x_{2 n+1}, p, t\right)\right\} \\
=\min \left\{F\left(y_{2 n}, y_{2 n+1}, p, q t\right),\right. \\
\left.F\left(y_{2 n+1}, y_{2 n+2}, p, q t\right)\right\}
\end{array}
$$

implies $F\left(y_{2 n+1}, y_{2 n+2}, p, q t\right) \geq F\left(y_{2 n}, y_{2 n+1}, p, t\right)$, because $F$ is nondecreasing. that

Also, letting $x=x_{2 n+2}$ and $y=x_{2 n+1}$ in (2.3), we have

$$
\begin{array}{r}
F\left(y_{2 n+2}, y_{2 n+3}, p, q t\right) \geq F\left(y_{2 n+1}, y_{2 n+2}, p, t\right) \\
\forall p \in X, t>0 .
\end{array}
$$

In general, we have

$$
F\left(y_{n}, y_{n+1}, p, q t\right) \geq F\left(y_{n-1}, y_{n}, p, t\right) .
$$

Thus for all $p \in X, t>0$ and $n=1,2,3 \ldots$

$$
F\left(y_{n}, y_{n+1}, p, t\right) \geq F\left(y_{0}, y_{1}, p, \frac{t}{q^{n}}\right)
$$

We now show that $\left\{y_{n}\right\}$ is a Cauchy sequence in $X$.

Let $\epsilon \in(0,1)$ be given. Since the $t$-norm $\Delta$ is of $H$ type, there exists $\lambda \in(0,1)$ such that for all $m, n \in N$ with $m>n$

$$
\Delta^{2^{m-n}}(1-\lambda)>1-\epsilon
$$

Since $\lim _{n \rightarrow \infty} F\left(y_{0}, y_{1}, p, t / q^{n}\right)=1$, there exists $n_{0} \in N$ such that for all $p \in X$ and $t>0, F\left(y_{0}, y_{1}, p, t / q^{n}\right)>1-\lambda$ for all $n \geq n_{0}$.

From (10) we have for all $p \in X$ and $t>0$, $F\left(y_{n}, y_{n+1}, p, t\right)>1-\lambda$ for all $n \geq n_{0}$.

Let $m>n \geq n_{0}$. Then for all $p \in X$ and $t>0$, we have

$$
\begin{aligned}
& F\left(y_{m}, y_{n}, p, t\right) \\
&= F\left(y_{m}, y_{n}, p, \frac{t}{3}+\frac{t}{3}+\frac{t}{3}\right) \\
& \geq \Delta\left(F\left(y_{m}, y_{n}, y_{n+1}, \frac{t}{3}\right),\right. \\
&\left.F\left(y_{m}, y_{n+1}, p, \frac{t}{3}\right), F\left(y_{n+1}, y_{n}, p, \frac{t}{3}\right)\right) \\
& \geq \Delta\left(F\left(y_{n+1}, y_{n}, p, \frac{t}{3}\right),\right.\left.\Delta\left(F\left(y_{n+1}, y_{n}, y_{m}, \frac{t}{3}\right), F\left(y_{n+1}, y_{m}, p, \frac{t}{3}\right)\right)\right) \\
& \geq \Delta\left(\Delta^{2}\left((1-\lambda), F\left(y_{n+1}, y_{m}, p, \frac{t}{3}\right)\right)\right) .
\end{aligned}
$$

Since

$$
\begin{array}{r}
F\left(y_{n+1}, y_{m}, p, \frac{t}{3}\right) \geq \Delta\left(F\left(y_{n+2}, y_{n+1}, p, \frac{t}{3^{2}}\right),\right. \\
\Delta\left(F\left(y_{n+2}, y_{n+1}, y_{m}, \frac{t}{3^{2}}\right),\right. \\
\left.\left.F\left(y_{n+2}, y_{m}, p, \frac{t}{3^{2}}\right)\right)\right) .
\end{array}
$$

From (13), we get

$$
F\left(y_{m}, y_{n}, p, t\right) \geq \Delta\left(\Delta^{2^{2}}(1-\lambda), F\left(y_{n+2}, y_{m}, p, \frac{t}{3^{2}}\right)\right) \text {. }
$$

Inductively, we obtain

$$
\begin{aligned}
F\left(y_{m}, y_{n}, p, t\right) & \geq \Delta\left(\Delta^{2^{m-n}}(1-\lambda), F\left(y_{m}, y_{m}, p, \frac{t}{3^{m-n}}\right)\right) \\
& =\Delta^{2^{m-n}}(1-\lambda) .
\end{aligned}
$$

From (11) and (13) we get, for all $p \in X$ and $t>$ $0, F\left(y_{m}, y_{n}, p, t\right)>1-\epsilon$ for all $m>n \geq n_{0}$. Thus $\left\{y_{n}\right\}$ is 
a Cauchy sequence in $X$. Since $X$ is complete, there exists a point $z$ in $X$ such that $\lim _{n \rightarrow \infty} y_{n}=z$ and this gives

$$
\begin{aligned}
\lim _{n \rightarrow \infty} S x_{2 n} & =\lim _{n \rightarrow \infty} T x_{2 n-1}=\lim _{n \rightarrow \infty} A x_{2 n-2} \\
& =\lim _{n \rightarrow \infty} B x_{2 n-1}=z \quad \forall n \in N .
\end{aligned}
$$

Without loss of generality, we assume that $S(X)$ is a complete subspace of $X$; therefore, $z=S u$ for some $u \in X$.

Subsequently, we have

$$
\lim _{n \rightarrow \infty} S x_{n}=\lim _{n \rightarrow \infty} T x_{n}=\lim _{n \rightarrow \infty} A x_{n}=\lim _{n \rightarrow \infty} B x_{n}=z=S u .
$$

Next, we claim that $A u=S u$. have

For this purpose, we put $x=u$ and $y=x_{n}$ in (2.3); we

$$
\begin{aligned}
& F\left(A u, B x_{n}, p, q t\right) \\
& \geq \min \left\{F\left(S u, T x_{n}, p, t\right), F(A u, S u, p, t),\right. \\
& \left.F\left(B x_{n}, T x_{n}, p, t\right), F\left(A u, T x_{n}, p, t\right)\right\} .
\end{aligned}
$$

Taking limit as $n \rightarrow \infty$

$$
\begin{gathered}
F(A u, z, p, q t) \geq \min \{F(z, z, p, t), F(A u, z, p, t), \\
F(z, z, p, t), F(A u, z, p, t)\} \\
\geq F(A u, z, p, t) .
\end{gathered}
$$

By Lemma 13, we have $A u=z$.

Hence $A u=S u=z$.

Since $A(X) \subset T(X)$, there exists a point $v \in X$ such that $A u=z=T v$.

Next we claim that $T v=B v$.

Putting $x=u$ and $y=v$ in (2.3), we have

$F(A u, B v, p, q t) \geq \min \{F(S u, T v, p, t), F(A u, S u, p, t)$,

$$
\begin{aligned}
& \quad F(B v, T v, p, t), F(A u, T v, p, t)\} \\
& \geq \min \{F(z, z, p, t), F(z, z, p, t), \\
& F(B v, T v, p, t), F(z, z, p, t)\} \\
& \geq F(B v, T v, p, t) .
\end{aligned}
$$

That is, $F(T v, B v, p, q t) \geq F(B v, T v, p, t)$.

By Lemma 13, we have $T v=B v$.

Thus $A u=S u=T v=B v=z$.

Since the pairs $(A, S)$ and $(B, T)$ are weakly compatible and $u$ and $v$ are their points of coincidence, respectively, then

$$
\begin{aligned}
& A z=A(S u)=S(A u)=S z, \\
& B z=B(T v)=T(B v)=T z .
\end{aligned}
$$

Now we prove that $z$ is a common fixed point of $A, B, S$, and $T$.
For this purpose, puting $x=z$ and $y=v$ in (2.3), we get

$$
\begin{array}{r}
F(A z, B v, p, q t) \geq \min \{F(S z, T v, p, t), F(A z, S z, p, t), \\
F(B v, T v, p, t), F(A z, T v, p, t)\} \\
\geq \min \{F(A z, B v, p, t), F(A z, A z, p, t), \\
F(B v, B v, p, t), F(A z, B v, p, t)\} \\
\geq F(A z, B v, p, t) .
\end{array}
$$

By Lemma 13, we have $A z=B v=z$.

Hence $z=A z=S z$, and $z$ is a common fixed point of $A$ and $S$. One can prove that $B v=z$ is also a common fixed point of $B$ and $T$.

Uniqueness. Suppose $z \neq w$ is another fixed point of $A, B, S$, and $T$.

Then, for all $p \in X$ with $p \neq z$ and $p \neq w$ and $t>0$,

$$
\begin{aligned}
& F(z, w, p, q t)= F(A z, B w, p, q t) \\
& \geq \min \{F(S z, T w, p, t), F(A z, S z, p, t), \\
&F(B w, T w, p, t), F(A z, T w, p, t)\} \\
& \geq \min \{F(z, w, p, t), F(z, z, p, t), \\
&F(w, w, p, t), F(z, w, p, t)\} \\
& \geq F(z, w, p, t),
\end{aligned}
$$

which implies that $F(z, w, p, q t) \geq F(z, w, p, t)$.

Hence $z=w$ is a unique common fixed point of $A, B, S$, and $T$.

This completes the proof of the theorem.

Corollary 15. Let $(X, F, \Delta)$ be a 2-Menger space with continuous t-norm $\Delta$ of $H$ type. Let $S$ and $T$ be self-mappings of $X$. Then $S$ and $T$ have a unique common fixed point in $X$ if and only if there exist two self-mappings $A, B$ of $X$ satisfying the following:

(2.5) $A(X) \subset S(X)$;

(2.6) pair $(A, S)$ is weakly compatible;

(2.7) there exists $q \in(0,1)$ such that for every $x, y, p \in X$ and $t>0$

$$
\begin{array}{r}
F(A x, A y, p, q t) \\
\geq \min \{F(S x, S y, p, t), F(A x, S x, p, t), \\
F(A y, S y, p, t), F(A x, S y, p, t)\} ;
\end{array}
$$

(2.8) one of the subspaces $A(X)$ or $S(X)$ is a closed subspace of $X$.

Indeed, $A$ and $S$ have a unique common fixed point in $X$.

Proof. We can easily prove the theorem by setting $B=A$ and $T=S$, in the proof of the Theorem 14 . 
Corollary 16. Let $(X, F, \Delta)$ be a 2-Menger space with continuous $t$-norm $\Delta$ of $H$ type. Let $S$ and $T$ be self-mappings of $X$. Then $S$ and $T$ have a unique common fixed point in $X$ if and only if there exist two self-mappings $A, B$ of $X$ satisfying (2.1), (2.2), (2.4), and the following:

(2.9) there exists $q \in(0,1)$ such that for every $x, y, p \in X$ and $t>0, F(A x, B y, p, q t) \geq F(S x, T y, p, t)$.

Indeed, $A, B, S$, and Thave a unique common fixed point in $X$.

Proof. Proof easily follows by setting $\min \{F(S x, T y, p, t)$, $F(A x, S x, p, t), \quad F(B y, T y, p, t), \quad F(A x, T y, p, t)\}=$ $F(S x, T y, p, t)$ in the proof of Theorem 14 .

\section{E.A. Property and Weakly Compatible Maps}

Now we prove our main result for weakly compatible maps along with the E.A. property as follows.

Theorem 17. Let $(X, F, \Delta)$ be a 2-Menger space with continuous t-norm $\Delta$ of $H$ type. Let $A, B, S$, and $T$ be self-mappings of $X$ satisfying (2.1), (2.2), (2.3), and (2.4) and the following:

(3.1) pair $(A, S)$ or pair $(B, T)$ satisfies E.A. property.

Then $A, B, S$, and $T$ have a unique common fixed point in $X$.

Proof. If the pair $(B, T)$ satisfies the property E.A., then there exists a sequence $\left\{x_{n}\right\}$ in $X$ such that $\lim _{n \rightarrow \infty} B x_{n}=$ $\lim _{n \rightarrow \infty} T x_{n}=z$ for some $z \in X$.

Since $B(X) \subset S(X)$, there exists a sequence $\left\{y_{n}\right\}$ in $X$ such that $B x_{n}=S y_{n}$. Hence $\lim _{n \rightarrow \infty} S y_{n}=z$. Also $A(X) \subset T(X)$; there exists a sequence $\left\{y_{n}^{\prime}\right\}$ in $X$ such that $A y_{n}^{\prime}=T x_{n}$. Hence $\lim _{n \rightarrow \infty} A y_{n}^{\prime}=z$.

Suppose that $S(X)$ is a complete subspace of $X$. Then $z=$ Su for some $u \in X$; subsequently; we have

$$
\begin{aligned}
\lim _{n \rightarrow \infty} B x_{n} & =\lim _{n \rightarrow \infty} T x_{n}=z=\lim _{n \rightarrow \infty} A y_{n}^{\prime} \\
& =\lim _{n \rightarrow \infty} S y_{n}=z=S u \quad \text { for some } u \in X .
\end{aligned}
$$

Next, we claim that $A u=S u$. gives

For this purpose, we put $x=u$ and $y=x_{n}$ in (2.3); this

$$
\begin{aligned}
& F\left(A u, B x_{n}, p, q t\right) \\
& \geq \min \left\{F\left(S u, T x_{n}, p, t\right), F(A u, S u, p, t),\right. \\
& \left.F\left(B x_{n}, T x_{n}, p, t\right), F\left(A u, T x_{n}, p, t\right)\right\} .
\end{aligned}
$$

Taking limit as $n \rightarrow \infty$

$$
\begin{aligned}
F(A u, z, p, q t) & \\
\geq \min \{ & F(z, z, p, t), F(A u, z, p, t), \\
& F(z, z, p, t), F(A u, z, p, t)\} \\
\geq & F(A u, z, p, t) .
\end{aligned}
$$

By Lemma 13, we have $A u=z$.

Hence $A u=S u=z$.

Since $A(X) \subset T(X)$, there exists a point $v \in X$ such that $A u=z=T v$.

Next we claim that $T v=B v$.

Putting $x=u$ and $y=v$ in (2.3), we have

$$
\begin{gathered}
F(A u, B v, p, q t) \\
\geq \min \{F(S u, T v, p, t), F(A u, S u, p, t), \\
F(B v, T v, p, t), F(A u, T v, p, t)\} \\
\geq \min \{F(z, z, p, t), F(z, z, p, t), \\
F(B v, T v, p, t), F(z, z, p, t)\} \\
\geq F(B v, T v, p, t) .
\end{gathered}
$$

That is, $F(T v, B v, p, q t) \geq F(B v, T v, p, t)$.

By Lemma 13, we have $T v=B v$.

Thus $A u=S u=T v=B v=z$.

Since the pairs $(A, S)$ and $(B, T)$ are weakly compatible and $u$ and $v$ are their points of coincidence, respectively, then $A z=A(S u)=S(A u)=S z$ and $B z=B(T v)=T(B v)=T z$.

Now we prove that $z$ is a common fixed point of $A, B, S$, and $T$.

For this purpose, we put $x=z$ and $y=v$ in (2.3); we get

$$
\begin{array}{r}
F(A z, B v, p, q t) \\
\geq \min \{F(S z, T v, p, t), F(A z, S z, p, t), \\
F(B v, T v, p, t), F(A z, T v, p, t)\} \\
\geq \min \{F(A z, B v, p, t), F(A z, A z, p, t), \\
F(B v, B v, p, t), F(A z, B v, p, t)\} \\
\geq F(A z, B v, p, t) .
\end{array}
$$

By Lemma 13, we have $A z=B v=z$.

Hence $z=A z=S z$ and $z$ is a common fixed point of $A$ and $S$. One can prove that $B v=z$ is also a common fixed point of $B$ and $T$.

Uniqueness. Suppose $z \neq w$ is another fixed point of $A, B, S$, and $T$.

Then, for all $p \in X$ with $p \neq z$ and $p \neq w$ and $t>0$,

$$
\begin{gathered}
F(z, w, p, q t) \\
=F(A z, B w, p, q t) \\
\geq \min \{F(S z, T w, p, t), F(A z, S z, p, t), \\
\quad F(B w, T w, p, t), F(A z, T w, p, t)\} \\
\geq \min \{F(z, w, p, t), F(z, z, p, t), \\
\quad F(w, w, p, t), F(z, w, p, t)\} \\
\geq F(z, w, p, t),
\end{gathered}
$$

which implies that $F(z, w, p, q t) \geq F(z, w, p, t)$. 
Hence $z=w$ is a unique common fixed point of $A, B, S$, and $T$.

Corollary 18. Let $(X, F, \Delta)$ be a 2-Menger space with continuous t-norm $\Delta$ of $H$ type. Let $A, B, S$, and $T$ be self-mappings of $X$ satisfying (2.1), (2.2), (2.4), and (2.9). $X$.

Then $A, B, S$, and $T$ have a unique common fixed point in

\section{CLR Property and Weakly Compatible Maps}

Now we prove our main result for weakly compatible maps along with the $\mathrm{CLR}_{S}$ property as follows.

Theorem 19. Let $(X, F, \Delta)$ be a 2-Menger space with continuous t-norm $\Delta$ of $H$ type. Let $A, B, S$, and $T$ be self mapping of $X$ satisfying (2.1), (2.2), (2.3), and the following:

(4.1) pair $(A, S)$ or pair $(B, T)$ satisfies $C L R_{S}$ property;

(4.2) one of the subspaces $A(X), B(X), S(X)$, or $T(X)$ is a closed subspace of $X$.

Then $A, B, S$, and $T$ have a unique common fixed point in $X$.

Proof. If the pair $(A, S)$ satisfies the $\mathrm{CLR}_{S}$ property then there exists a sequence $\left\{x_{n}\right\}$ in $X$ such that $\lim _{n \rightarrow \infty} A x_{n}=$ $\lim _{n \rightarrow \infty} S x_{n}=z$, where $z \in S(X)$. Therefore, there exists a point $u \in X$ such that $S u=z$. Since $T(X)$ is a closed subspace of $X$ and $A(X) \subset T(X)$, for each $\left\{x_{n}\right\} \subset X$ there corresponds a sequence $\left\{y_{n}\right\} \subset X$ such that $A x_{n}=T y_{n}$. $S(X)$.

Therefore, $\lim _{n \rightarrow \infty} T x_{n}=\lim _{n \rightarrow \infty} A x_{n}=z$, where $z \in$

Thus in all, we have

$$
\lim _{n \rightarrow \infty} A x_{n}=\lim _{n \rightarrow \infty} S x_{n}=\lim _{n \rightarrow \infty} T y_{n}=z .
$$

Now we are required to show that $\lim _{n \rightarrow \infty} B y_{n}=z$.

Putting $x=x_{n}$ and $y=y_{n}$ in (2.3), we get

$$
\begin{array}{r}
F\left(A x_{n}, B y_{n}, p, q t\right) \\
\geq \min \left\{F\left(S x_{n}, T y_{n}, p, t\right), F\left(A x_{n}, S x_{n}, p, t\right),\right. \\
\left.F\left(B y_{n}, T y_{n}, p, t\right), F\left(A x_{n}, T y_{n}, p, t\right)\right\} .
\end{array}
$$

Let $\lim _{n \rightarrow \infty} B y_{n}=l \neq z$ for $t>0$. Then taking limit as $n \rightarrow \infty$, we have

$$
\begin{aligned}
F(z, l, p, q t) \geq \min \{F(z, z, p, t), F(z, z, p, t), \\
F(l, z, p, t), F(z, z, p, t)\} \\
\geq F(l, z, p, t) .
\end{aligned}
$$

By Lemma 13, we have $z=l$, then $\lim _{n \rightarrow \infty} B y_{n}=z$. Hence $\lim _{n \rightarrow \infty} A x_{n}=\lim _{n \rightarrow \infty} S x_{n}=\lim _{n \rightarrow \infty} T y_{n}=$ $\lim _{n \rightarrow \infty} B y_{n}=z=S u$, for some $u \in X$.

Uniqueness follows from Theorem 14.

Corollary 20. Let $(X, F, \Delta)$ be a 2-Menger space with continuous t-norm $\Delta$ of $H$ type. Let $A, B, S$, and $T$ be self-mappings of $X$ satisfying (2.1), (2.2), (2.9), (4.1), and (4.2). $X$.

\section{Application}

As an application of Theorem 14, we prove a common fixed point theorem for four finite families of mappings which runs as follows.

Theorem 21. Let $\left\{A_{1}, A_{2}, \ldots, A_{m}\right\},\left\{B_{1}, B_{2}, \ldots, B_{n}\right\},\left\{S_{1}, S_{2}\right.$, $\left.\ldots, S_{p}\right\}$, and $\left\{T_{1}, T_{2}, \ldots, T_{q}\right\}$ be four finite families of selfmappings of a 2-Menger space $(X, F, \Delta)$ with continuous $t$ norm of Hadzic type such that $A=A_{1} A_{2} \cdots A_{m}, B=$ $B_{1} B_{2} \cdots B_{n}, S=S_{1} S_{2} \cdots S_{p}$, and $T=T_{1} T_{2} \cdots T_{q}$ satisfy condition (2.1), (2.2), (2.3), and (2.4); then

(i) A and S have a point of coincidence;

(ii) B and T have a point of coincidence.

Moreover, if $A_{i} A_{j}=A_{j} A_{i}, B_{k} B_{l}=B_{l} B_{k}, S_{r} S_{s}=S_{s} S_{r}, T_{t} T_{u}=$ $T_{u} T_{t}, \quad A_{i} S_{r}=S_{r} A_{i}$, and $B_{k} T_{t}=T_{t} B_{k}$ for all $i, j \in I_{1}=$ $\{1,2, \ldots, m\}, k, l \in I_{2}=\{1,2, \ldots, n\}, r, s \in I_{3}=\{1,2, \ldots, p\}$, and $t, u \in I_{4}=\{1,2, \ldots, q\}$, then (for all $i \in I_{1}, k \in I_{2}, r \in I_{3}$, and $\left.t \in I_{4}\right) A_{i}, S_{r}, B_{k}$, and $T_{t}$ have a common fixed point.

Proof. The conclusions (i) and (ii) are immediate as $A, S, B$, and $T$ satisfy all the conditions of Theorem 14 . Now appealing to component wise commutativity of various pairs, one can immediately prove that $A S=S A$ and $B T=T B$, and hence, obviously both pairs $(A, S)$ and $(B, T)$ are coincidently commuting. Note that all the conditions of Theorem 14 (for mappings $A, S, B$, and $T$ ) are satisfied ensuring the existence of a unique common fixed point, say $z$. Now one needs to show that $z$ remains the fixed point of all the component maps. For this consider

$$
\begin{aligned}
A\left(A_{i} z\right) & =\left(\left(A_{1} A_{2} \cdots A_{m}\right) A_{i}\right) z \\
& =\left(A_{1} A_{2} \cdots A_{m-1}\right)\left(\left(A_{m} A_{i}\right) z\right) \\
& =\left(A_{1} A_{2} \cdots A_{m-1}\right)\left(A_{i} A_{m} z\right) \\
& =\left(A_{1} A_{2} \cdots A_{m-2}\right)\left(A_{m-1} A_{i}\left(A_{m} z\right)\right) \\
& =\left(A_{1} A_{2} \cdots A_{m-2}\right)\left(A_{i} A_{m-1}\left(A_{m} z\right)\right) \\
& =\cdots=A_{1} A_{i}\left(A_{2} A_{3} A_{4} \cdots A_{m} z\right) \\
& =A_{i} A_{1}\left(A_{2} A_{3} A_{4} \cdots A_{m} z\right)=A_{i}(A z)=A_{i} z .
\end{aligned}
$$

Similarly, one can show that

$$
\begin{array}{cc}
A\left(S_{r} z\right)=S_{r}(A z)=S_{r} z, & S\left(S_{r} z\right)=S_{r}(S z)=S_{r} z, \\
S\left(A_{i} z\right)=A_{i}(S z)=A_{i} z, & B\left(B_{k} z\right)=B_{k}(B z)=B_{k} z, \\
B\left(T_{t} z\right)=T_{t}(B z)=T_{t} z, \quad T\left(T_{t} z\right)=T_{t}(T z)=T_{t} z, \\
T\left(B_{k} z\right)=B_{k}(T z)=B_{k} z,
\end{array}
$$

which show that (for all $i, r, k$, and $t$ ) $A_{i} z$ and $S_{r} z$ are other fixed points of the pair $(A, S)$ whereas $B_{k} z$ and $T_{t} z$ are other fixed points of the pair $(B, T)$. Now appealing to the 
uniqueness of common fixed points of both pairs separately, we get

$$
z=A_{i} z=S_{r} z=B_{k} z=T_{t} z
$$

which shows that $z$ is a common fixed point of $A_{i}, S_{r}, B_{k}$, and $T_{t}$ for all $i, r, k$, and $t$.

By setting $A=A_{1}=A_{2}=\cdots=A_{m}, B=B_{1}=B_{2}=\cdots=$ $B_{n}, S=S_{1}=S_{2}=\cdots=S_{p}$, and $T=T_{1}=T_{2}=\cdots=T_{q}$, one deduces the following for certain iterates of maps, which run as follows.

Corollary 22. Let $A, B, S$, and $T$ be four self-mappings of a 2Menger space $(X, F, \Delta)$ such that $A^{m}, B^{n}, S^{p}$, and $T^{q}$ satisfy the conditions (2.1) and (2.3). If one of $A^{m}(X), B^{n}(X), S^{p}(X)$, or $T^{q}(X)$ is a closed subset of $X$, then $A, B, S$, and $T$ have a unique common fixed point provided $(A, S)$ and $(B, T)$ commute.

\section{Acknowledgments}

One of the authors (S. Kumar) would like to acknowledge the UGC for providing financial grant through Major Research Project under Ref. 39-41/2010 (SR). I am also thankful to the referee for careful reading of the paper and giving useful comments to improve it.

\section{References}

[1] V. M. Sehgal and A. T. Bharucha-Reid, "Fixed points of contraction mappings on probabilistic metric spaces," Mathematical Systems Theory, vol. 6, pp. 97-102, 1972.

[2] O. Hadžić, "On common fixed point theorems in 2-metric spaces," Univerzitet u Novom Sadu, Zbornik Radova PrirodnoMatematičkog Fakulteta, vol. 12, pp. 7-18, 1982.

[3] O. Hadžić, "Some theorems on the fixed points in probabilistic metric and random normed spaces," Bollettino dell'Unione Matematica Italiana B, vol. 1, no. 6, pp. 381-391, 1982.

[4] O. Hadžić, "Fixed point theorems for multivalued mappings in some classes of fuzzy metric spaces," Fuzzy Sets and Systems, vol. 29, no. 1, pp. 115-125, 1989.

[5] O. Hadzic, "A fixed point theorem for multivalued mappings in 2-Menger spaces," Univerzitet u Novom Sadu, Zbornik Radova Prirodno-Matematičkog Fakulteta, vol. 24, pp. 1-7, 1994.

[6] O. Hadžić, "A fixed point theorem in Menger spaces," Institut Mathématique, vol. 40, pp. 107-112, 1979.

[7] O. Hadžić and E. Pap, Fixed Point Theory in Probabilistic Metric Spaces, Kluwer Academic Publishers, Dordrecht, The Netherlands, 2001.

[8] T. L. Hicks, "Fixed point theory in probabilistic metric spaces," Univerzitet u Novom Sadu, Zbornik Radova PrirodnoMatematičkog Fakulteta, vol. 13, pp. 63-72, 1983.

[9] S. Gähler, "2-metrische Räume und ihre topologische Struktur," Mathematische Nachrichten, vol. 26, pp. 115-148, 1963.

[10] K. Iseki, "Fixed point theorems in 2-metric spaces," Mathematics Seminar Notes, vol. 3, pp. 133-136, 1975.

[11] B. G. Pachpatte, "Fixed point theorems for contraction type mappings on a 2-metric space," Proceedings of the National Academy of Sciences, India A, vol. 48, no. 2, pp. 94-102, 1978.
[12] B. E. Rhoades, "Contraction type mappings on a 2-metric space," Mathematische Nachrichten, vol. 91, pp. 151-155, 1979.

[13] S.-S. Chang and N. J. Huang, "On the generalized 2-metric spaces and probabilistic 2-metric spaces with applications to fixed point theory," Mathematica Japonica, vol. 34, no. 6, pp. 885-900, 1989.

[14] W. Z. Zeng, "Probabilistic 2-metric spaces," Journal of Mathematical Research and Exposition, vol. 7, no. 2, pp. 241-245, 1987.

[15] Y. Shi, L. Ren, and X. Wang, "The extension of fixed point theorems for set valued mapping," Journal of Applied Mathematics \& Computing, vol. 13, no. 1-2, pp. 277-286, 2003.

[16] G. Jungck, "Common fixed points for noncontinuous nonself maps on nonmetric spaces," Far East Journal of Mathematical Sciences, vol. 4, no. 2, pp. 199-215, 1996.

[17] M. Aamri and D. El Moutawakil, "Some new common fixed point theorems under strict contractive conditions," Journal of Mathematical Analysis and Applications, vol. 270, no. 1, pp. 181$188,2002$.

[18] M. Imdad and J. Ali, "Jungck's common fixed point theorem and E.A property," Acta Mathematica Sinica, vol. 24, no. 1, pp. 87-94, 2008.

[19] G. Jungck and B. E. Rhoades, "Fixed points for set valued functions without continuity," Indian Journal of Pure and Applied Mathematics, vol. 29, no. 3, pp. 227-238, 1998.

[20] H. K. Pathak, "Common fixed point theorems using property (E.A) in complex-valued metric spaces," Thai Journal of Mathematics. In press.

[21] W. Sintunavarat and P. Kumam, "Common fixed point theorems for a pair of weakly compatible mappings in fuzzy metric spaces," Journal of Applied Mathematics, vol. 2011, Article ID 637958, 14 pages, 2011.

[22] J. Han, "A common fixed point theorem on Fuzzy 2-metric spaces," Journal of the Chung Cheong Mathematical Society, vol. 23 , no. $4,2010$. 


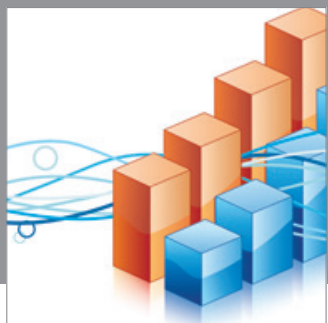

Advances in

Operations Research

mansans

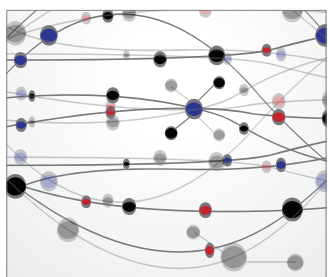

The Scientific World Journal
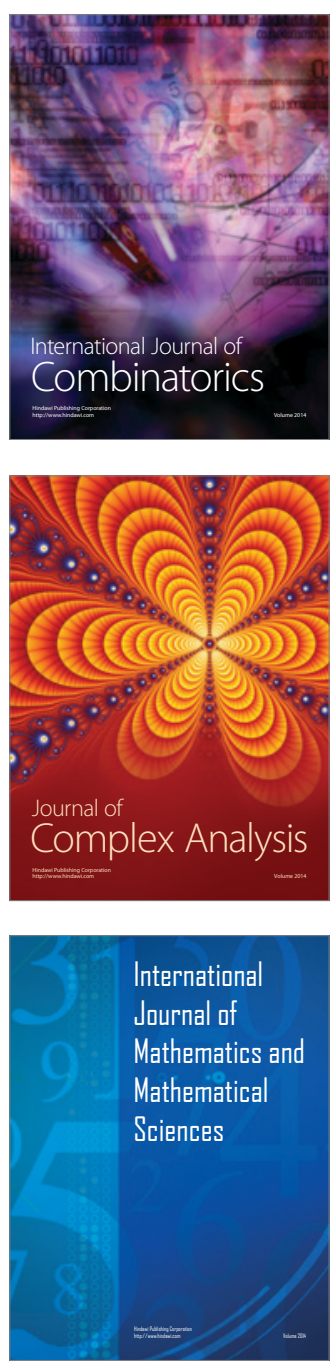
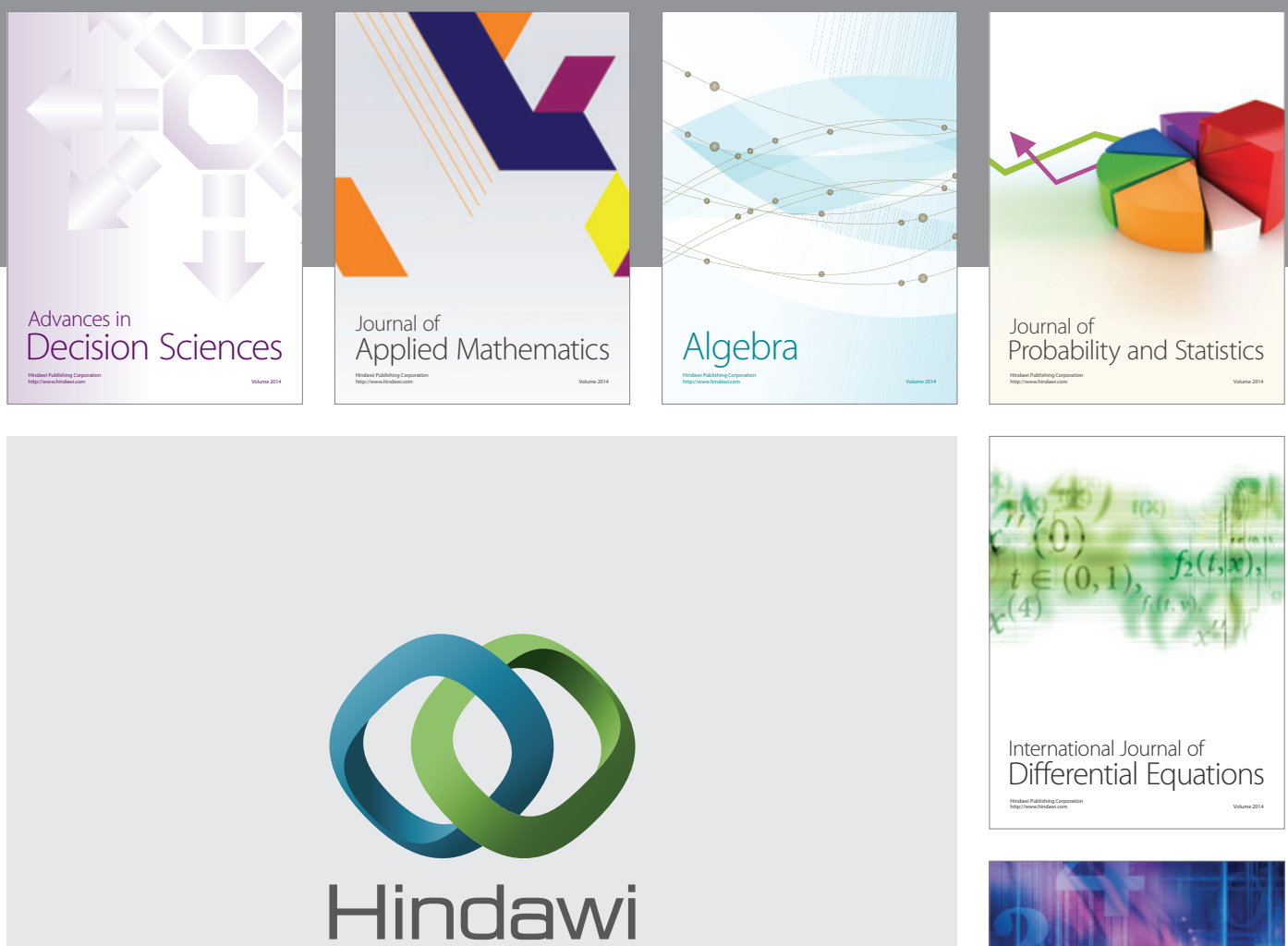

Submit your manuscripts at http://www.hindawi.com
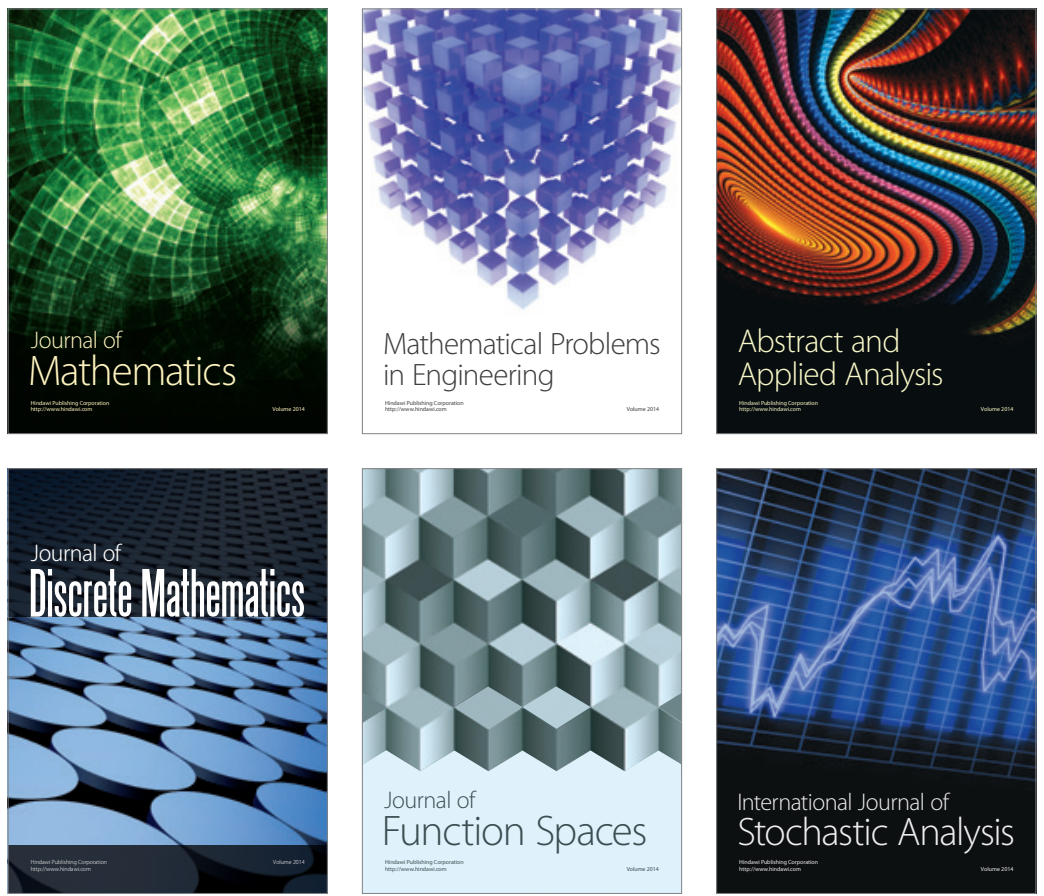

Journal of

Function Spaces

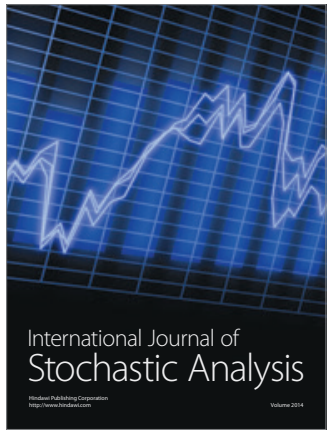

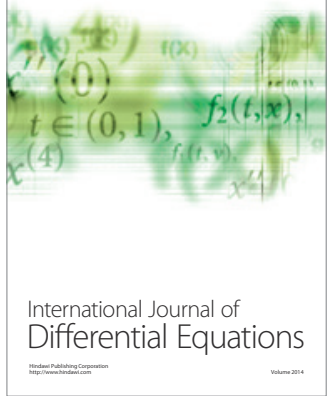
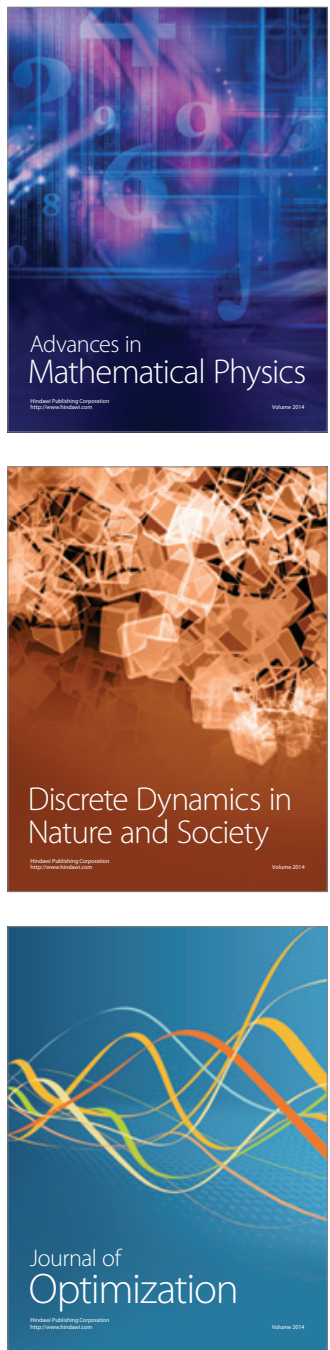